\title{
Chapter 41 \\ Exploring Genetic Resources to Increase Adaptation of Wheat to Climate Change
}

\author{
Matthew Reynolds, Maria Tattaris, C. Mariano Cossani, Marc Ellis, \\ Kazuko Yamaguchi-Shinozaki, and Carolina Saint Pierre
}

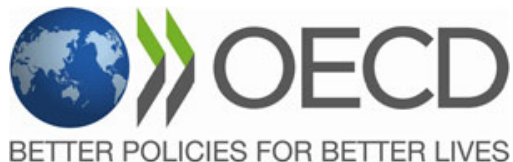

The opinions expressed and arguments employed in this publication are the sole responsibility of the authors and do not necessarily reflect those of the OECD or of the governments of its Member countries.The Special Session was sponsored by the OECD Co-operative Research Programme on Biological Resource Management for Sustainable Agricultural Systems, whose financial support made it possible for most of the invited speakers to participate in the Special Session.

\begin{abstract}
The combined problems of climate change, population growth, and increased demands on a declining natural resource base force scientists to push crop performance to its limits. A powerful strategy is to explore genetic resources to identify promising material that can be used directly in breeding, for gene discovery, and to further understand the mechanisms of adaptation. Initially traits must be defined for stress targets using conceptual models, examples being better root systems to access subsoil deep water and the ability to store and remobilize water soluble carbohydrates from storage tissue. New sources of diversity for such traits can be found in collections such as the World Wheat Collection housed at CIMMYT; for example, Mexican landraces provide good sources of both of these traits. Being a
\end{abstract}

M. Reynolds $(\bowtie) \bullet$ M. Tattaris $\bullet$ C.M. Cossani $\bullet$ M. Ellis $\bullet$ C.S. Pierre International Maize and Wheat Improvement Center (CIMMYT), Mexico, DF, Mexico e-mail: m.reynolds@cgiar.org

K. Yamaguchi-Shinozaki

Japan International Research Center for Agricultural Sciences (JIRCAS), Ibaraki, Japan 
polyploid, wheat has a useful secondary gene pool that can be used to re-synthesize hexaploid wheat, while transgenic approaches remove all taxonomic limits to plant improvement. To efficiently explore genetic resources, for crop improvement and to identify genetic and mechanistic bases, requires high throughput phenotyping approaches. For example, an airborne remote sensing platforms is used to determine spectral indices associated with temperature, water content, and pigment composition of leaves via thermal and multispectral imagery. Using the above approaches, best lines are used directly in pre-breeding to combine favorable combinations of traits and their alleles. These approaches have already delivered a new generation of drought adapted lines where cumulative gene action on yield is observed through strategic combination of stress adaptive traits many coming from landraces or products of wide crossing with wheat wild relatives.

Keywords Landraces $\bullet$ Physiological breeding $\bullet$ Phenotyping $\bullet$ Remote sensing $\bullet$ Synthetic wheat

\section{Introduction}

The CIMMYT coordinated International Wheat Improvement Network (IWIN) partners with hundreds of wheat breeders worldwide to provide new genotypes $(\sim 1,000$ annually) to national programs as international public goods, through the following mechanisms (Braun et al. 2010):

- Free exchange of germplasm with all national public and private breeding programs worldwide, including accessions from genetic resource collections.

- Centralized breeding hubs that focus on generic needs - i.e., yield potential, yield stability, genetic resistance to range of biotic and abiotic stresses, consumeroriented quality traits.

- Distribution of international nurseries specifically targeted to a number of major agro-ecosystems, via national wheat programs worldwide.

- Analysis of international yield trials and free access to all data collected.

- Global disease and pest monitoring to ensure relevance of current local, regional, and global breeding activities.

- Capacity building and training of research partners.

- Regular contact among research partners through consultation, workshops, etc., to help identify the latest technology needs.

Through the IWIN, wheat germplasm has spread continually since the Green Revolution and is now not only extremely well represented in farmers fields of the developing world, but is also commonly seen in the pedigrees of wheat lines in developed countries (Braun et al. 2010). The continued effectiveness of these wheat 
breeding strategies have been demonstrated right up the present with yield data from hundreds of testing sites worldwide showing average genetic yield gains of 1 $\%$ every year in germplasm targeted to water limited environments (Manes et al. 2012), and $0.6 \%$ per year in fully irrigated environments (Sharma et al. 2012) based on lines distributed since 1995. Germplasm distributed by IWIN is maintained resistant to the full range of diseases that commonly affect wheat (Braun et al. 2010). As a result, resource poor farmers in LDCs are buffered financially from having to apply expensive fungicide, while the environment is protected from the additional agrochemical burden. An important spin off of the IWIN are massive phenotypic datasets that have allowed breeders to identify germplasm with either specific adaptation to local challenges and diseases, or broad (spatial and/or temporal) adaptation to many locations and cropping systems (e.g., Gourdji et al. 2012).

In spite of this achievement, genetic gains still fall short of meeting the predicted demands by 2050 (Rosegrant and Agcaoili 2010), a mismatch that represents a serious challenge for future food security especially in the light of the challenges associated with climate change. One of the best ways to address this challenge from the point of view of genetic improvement is through a more systematic use of genetic resources in breeding. To achieve this requires a series of steps that are outlined in the rest of this paper.

\section{Identify Crop Characteristics Conferring Stress Adaptation}

Many crop characteristics have been reported as conferring improved stress adaptation (e.g., Rebetzke et al. 2009; Cossani and Reynolds 2012) and well controlled phenotypic studies can indicate whether these traits may be complementary in different combinations (Reynolds et al. 2007a). However, the only way to confirm their value definitively is to find sources with good expression of the traits and introgress into elite backgrounds. The approach has been successful within the IWIN for producing a new generation of drought adapted lines as will be discussed in the last section. Some of the traits that have proven to be most useful for both heat and drought adaption include (see Richards 2006; Rebetzke et al. 2009; Reynolds et al. 2010):

- Cooler canopy temperature that indicates access to subsoil water under drought and an adequate vascular system -including roots- to match evaporative demand at high vapour pressure deficit typical of heat stress in dry environments.

- Rapid early ground cover to avoid wasteful evaporation of water at the soil surface back to the atmosphere.

- Intrinsic transpiration efficiency that ensures conservative use of water when it is relatively abundant as a water budgeting strategy. 
- Epicuticular leaf wax that reflects excess radiation and reduces evaporation from the leaf surface thereby reducing the risk of photo-inhibition and dehydration, respectively.

- Accumulation and remobilization of water soluble carbohydrates in storage organs like stems permitting grain-filling to continue even when post-anthesis stress is too severe to permit adequate carbon assimilation.

- Membrane thermo-stability that can be screened using electrolyte leakage, and chlorophyll fluorescence that, although it has not been used systematically in selecting parents, has become a recent focus for screening.

\section{Exploration of Genetic Resources for Adaptive Traits: Landraces}

CIMMYT and other wheat breeding programs have been using landraces to broaden the wheat gene pool for decades (Smale et al. 2002), mostly for disease resistance traits as these are relatively easy to detect in un-adapted material and select for in segregating progeny (Reynolds and Borlaug 2006). More recently Mexican landraces were collected -after approximately 500 years of essentially natural selection in some of Mexico's harshest rain-fed environments-. These were screened for heat and drought adaptive traits and a few of the lines show exceptional characteristics such as deep roots and ability to store water soluble carbohydrates in the stem (Reynolds et al. 2007b). Very recently, over 70,000 accessions of the World Wheat Collection have been screened for adaptation to heat and drought stress -mostly for the first time-, and diverse panels have been assembled for genetic analysis. These include elite durum and bread wheat lines, landraces from hot and dry regions worldwide, and lines derived from interspecific hybridization with wheat's relatives, including 're-synthesized' wheat (see next section).

Other more targeted approaches include characterization of panels identified using agro-ecological data also known as the Focused Identification of Germplasm Strategy or FIGS (http://www.figs.icarda.net/). The principle is that landraces from heat and drought stressed regions are more likely to contain stress adaptive-traits. In the case of drought, we obtained 204 landraces originating from drought-affected areas. These were grown in Cd Obregon, Sonora, under drought conditions (only one irrigation at sowing providing approximately $180 \mathrm{~mm}$ of available water to $1.2 \mathrm{~m}$ depth, and no significant rainfall during the growing season). CIMMYT varieties were included as checks. Total above ground biomass was estimated and grain yield after threshing (Fig. 41.1).

As shown in Fig. 41.1, lines capable of producing high biomass were identified. Over $45 \%$ of the entries had greater biomass under drought than the CIMMYT check varieties, some with $40 \%$ greater biomass. The harvest index of the lines was lower than that of CIMMYT checks and as a result only $5 \%$ of the entries had 

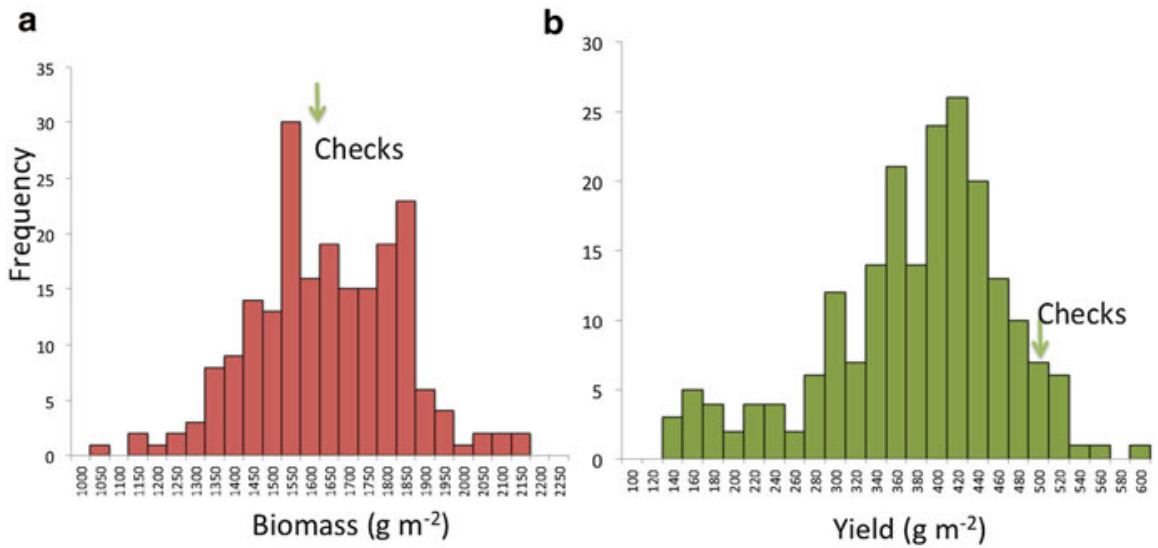

Fig. 41.1 Frequency distribution of 208 FIGS lines grown under drought for (a) total biomass (b) grain yield. The mean of three CIMMYT checks (Sokoll, Roelfs, Weebil) is indicated by the green arrow

greater yield than the CIMMYT varieties. This is not unexpected for landraces: most of the FIGS lines were tall, and some were late-flowering resulting in greater stress during grain filling. These traits would have contributed to the low harvest index of the landraces in addition to not necessarily being adapted to the photoperiod conditions of the experimental environment. The lines that demonstrated the capacity to produce high biomass are being crossed to CIMMYT elite lines as part of the Physiology Group's pre-breeding program. The progeny lines will be selected for semi-dwarf stature and appropriate maturity.

\section{Inter-specific Hybridization to Broaden the Crop Gene Pool}

Although the gene pools used in conventional breeding are relatively restricted (Hajjar and Hodgkin 2007), a vast pool of genetic resources is available for breeding. Different gene pools have been defined depending on the difficulty of employing them. The most easy to use are those from the primary gene pool represented by germplasm that share a common genome but which have become isolated from mainstream gene pools such as landraces (as discussed in the section above). The secondary gene pool is represented by closely-related genomes that can be utilized through inter-specific hybridization, and would include the development of socalled "synthetic" or "re-synthesized" wheat, where tetraploid durum wheat has been hybridized with Aegilops tauschii, the ancestral donor of the D-genome, to recreate hexaploid bread wheat (Mujeeb-Kazi et al. 1996). This approach has been successful in introducing disease resistance as well as drought adaptive traits 

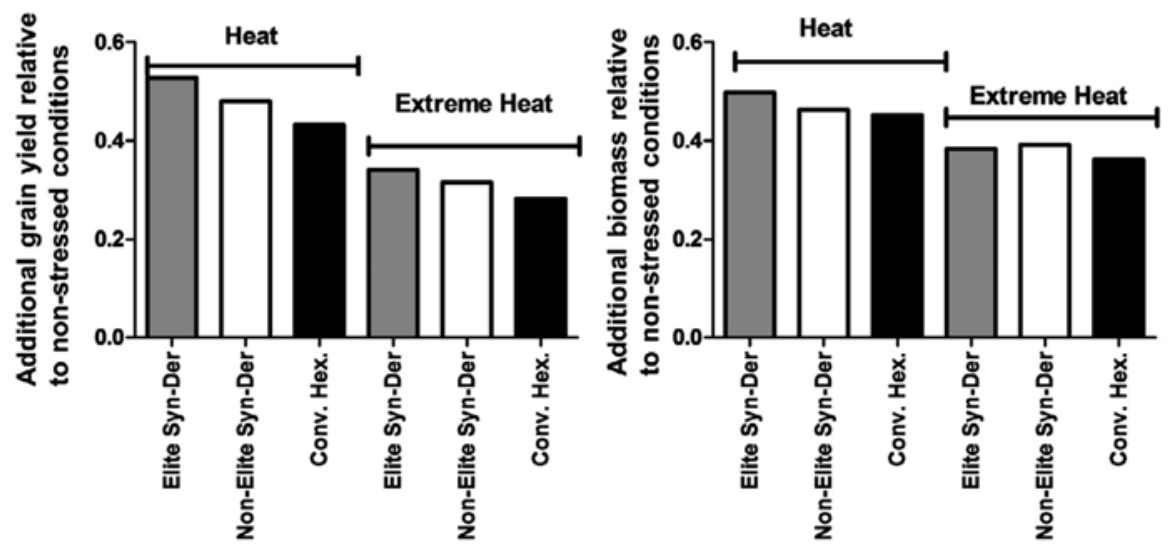

Fig. 41.2 Yield and biomass of synthetic hexaploid wheat derived lines under two different late sown environments expressed as additional yield (left) or biomass (right) relative to non-stressed conditions, NW Mexico, 2012-2013

(Reynolds et al. 2007b; Trethowan and Mujeeb-Kazi 2008). Many thousands of accessions of both ancestral genomes (AB and D) exist as candidates for interspecific hybridization. Physiological characterization is being used to help select the most promising accessions, as well as to select among the initial products (primary 'synthetics'), for pre-breeding and genetic studies. In parallel to this, genotyping of existing primary synthetics and potential progenitors (AB tetraploid, and Ae. tauschii) is being carried out to target under-utilized genetic diversity.

Analysis of impacts from using synthetic wheat in breeding have shown significant contributions to drought adaptation (Lopes and Reynolds 2011), and it appears that the introduction of alleles from the wild D genome have increased the capacity of roots to partition resources to lower soil profiles when experiencing water stress (Reynolds et al. 2007b). A recent analysis looked at the same lines under heat stress in the hot desert of NW Mexico. To generate moderate and more extreme heat stress in the field, fully irrigated crops were sown later than normal in the months of February and March, respectively (normal sowing occurring in December). Results showed highly significant impacts of synthetic derived (SYN-DER) lines in hot, irrigated environments on both yield and biomass (Fig. 41.2).

In general, and across all three environments (temperate, heat and extreme heat), the elite SYN-DER lines (primary synthetic lines backcrossed to a conventional wheat line) showed superiority in terms of performance over lines derived from single crosses to a conventional line or the conventional line itself. However, yield advantages of elite SYN-DER lines over conventional hexaploids were larger in both heat environments than under temperate environments. Biomass at maturity of the elite-SYN-DER lines was also higher than conventional hexaploid lines. The advantages in aboveground biomass were also observed at heading. The SYN-DER 
lines also showed larger expression than conventional lines for water soluble carbohydrates in stems and tended to be earlier maturing. These results underline the value of exploring the secondary gene pool of wheat.

\section{Transgenic Approaches}

Transgenic approaches remove all taxonomic limits to plant improvement. The manipulation of genes through biotechnology has provided the opportunity of genetic engineering plant responses to abiotic stresses such as heat, drought, and salinity (Qin et al. 2011). The regulated expression of stress-induced transcription factors, for example, has been identified as an attractive tool for improving stress tolerance, since transcription factors can regulate the expression of a large number of relevant downstream genes associated to abiotic stress responses in genetically modified plants (Nakashima et al. 2009). Transcription factors such as DREB1/ CBF, DREB2, AREB/ABF, and NAC, are used to improve stress tolerance to abiotic stresses in various grasses including wheat and rice.

Recent efforts to test the functionality of various transcription factors involved in complex physiological responses and to evaluate the effect of these under either constitutive or inducible promoters have been made in wheat. Transgenic wheat lines were developed at CIMMYT using new constructs generated at the Japan International Research Center for Agricultural Sciences (JIRCAS) and Rikagaku Kenkyūjo (RIKEN). The resulting lines were then characterized under glasshouse conditions and open field trials conducted at CIMMYT's subtropical experimental station in Tlaltizapán, Morelos, Mexico ( $18^{\circ} 41^{\prime} \mathrm{N}, 99^{\circ} 10^{\prime} \mathrm{W}, 940 \mathrm{~m}$ asl). In these open-field trials, physiological traits and grain yield performance of 116 homozygous low copy number GM wheat lines (14 gene-promoter combinations) were evaluated under different water regimes. The lines evaluated in the field showed no pleiotropic effect, nor unpredictable unwanted effects when compared to control lines (Saint Pierre et al. 2012). Based on a 3-year analysis, the most promising lines showed an average increase of yield under drought of $10 \%$ when compared to nontransformed line (var. Fielder).

Even though promising results have been observed, several challenges have yet to be overcome before anticipating a high impact of genetically modified lines on wheat production. Efforts need to address the identification of appropriate gene and gene-promoter combinations, the insertion of the proper transgene in appropriate backgrounds, and field selection strategies. Regulation processes, commercialization, and marketing of genetically modified products are parallel challenges to face. From the technical side, a critical step in the case of genetically modified wheat is that more efficient transformation protocols as well as precise expression systems would need to be defined. The wheat variety Fielder is commonly used in transformation due to its good embryogenesis capacity and regeneration efficiency; however it has lower yield potential than modern elite wheat lines. Then, after insertion 


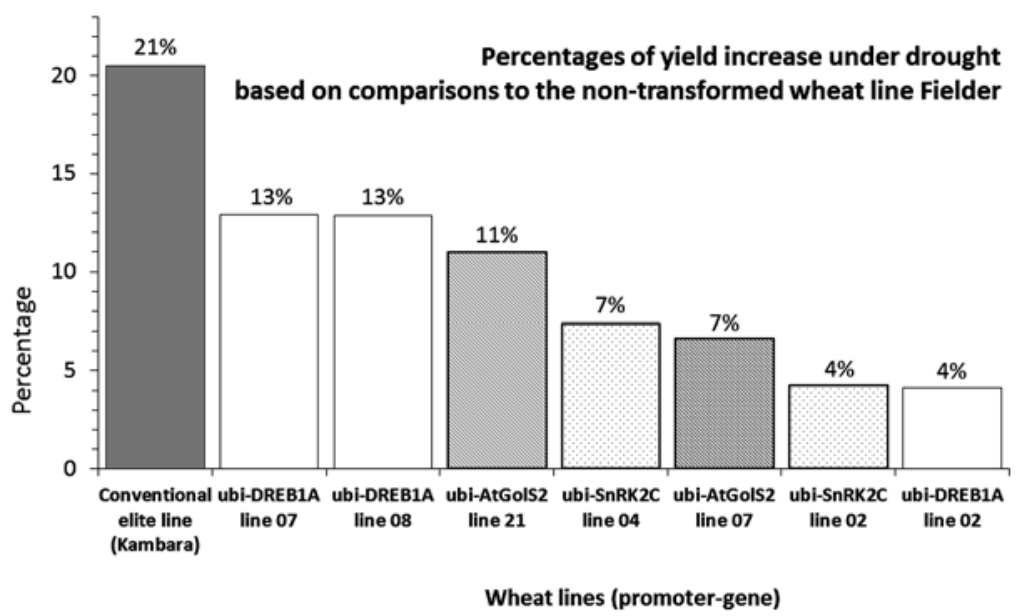

Fig. 41.3 Yield increases as percentage over non-transformed Fielder line, observed for the most promising lines from transformations using different gene-promoter combinations. Field trials were grown during 3-year, in open field trial in Mexico, 2010-2013

of the transgenes in wheat backgrounds (Fielder) it is necessary to transfer them to modern elite lines, which is currently achieved by backcrossing. Predictions based on extrapolating yield increases from the transformed line Fielder to new high performing germplasm (elite lines) should be made with caution. The challenge is, therefore, to break the yield barriers in modern elite wheat lines to finally achieve a significant improvement in wheat grain yield under stress. Collective and cooperative interventions from molecular biologists, physiologists, and breeders are required (Fig. 41.3).

\section{High Throughput and Precision Phenotyping}

The efficient exploration of genetic resources -for crop improvement and to identify genetic and mechanistic bases- requires a combination of precision and high throughput phenotyping approaches. Non-intrusive high spatial resolution spectral imagery can be applied to the monitoring of physiological characteristics such as canopy temperature, hydration status, and pigment composition, as well as permitting estimates of agronomic traits such as biomass and yield. Spaceborne remote sensing platforms have proven efficient at measuring some of these characteristics at a field scale, however their spatial resolution proves too low for accurate data retrieval at plot level in a plant breeding context. While ground-based remote sensing is used for predicting physiological and agronomic traits at a plot scale, 
temporal variations of environmental variables, such as air temperature, can introduce confounding factors, especially when applied to large trials. Low-level airborne remote sensing platforms overcome these restrictions, allowing for fast, non-destructive screening of plant physiological properties over large areas, with enough resolution to obtain information at plot level while being able to measure thousands genetic resources in the field with one take.

Rapid advances in remote sensing technologies, data processing and availability of instruments has made it easier to implement remote sensing techniques to research numerous plant properties (e.g., Leinonen and Jones 2004; Jones et al. 2007; Berni et al. 2009). The increase in demand for large scale vegetation monitoring means that there is a move to such remote sensing applications in which simultaneous measurements of greater target areas can readily be made. In a recent study, Tattaris et al. (2013) applied a low level airborne remote sensing platform to derive indices relating to plant properties such as canopy temperature, water status, and pigment composition. Sampling was performed with a multispectral camera and thermal camera mounted on an eight rotor unmanned aerial vehicle (UAV) and helium filled tethered blimp. Airborne indices were validated by equivalent indices collected at ground level. These ground-based measurements have already been proven to be linked with yield and biomass (e.g., Reynolds et al. 1994; Aparicio et al. 2000). This strong agreement between methods acts to validate the use of the airborne indices. In addition, significant genetic correlations were found between the airborne indices derived using imagery and yield/biomass, larger than the equivalent correlations between the ground-based measurements and yield/biomass. This is probably because airborne approaches reduce error in two ways. Firstly, simultaneous measurement permitted through airborne platforms avoid the confounding effects associated with environmental drift when measuring plots one at a time with ground-based instruments. Secondly, aerial imaging techniques permit data smoothing through elimination of outlier pixels. These factors give additional confidence to the use of such methodologies in screening genetic resources and breeding progeny at a large scale where thousands of plots are involved.

\section{Strategic Crossing to Achieve Cumulative Gene Action}

Trait selection has made continual progress in wheat breeding through incorporating agronomic traits such as height and flowering time, resistance to a spectrum of prevalent diseases, quality parameters determined by end use, and yield based on multi-location trials (Braun et al. 2010). However, current genetic gains are not adequate to meet future demand while climate change threatens to erode these further. Breeding more specifically for heat and drought adaption can help to increase genetic gains (Reynolds et al. 2009). The main objective of strategic trait-based crossing is to accumulate traits that will be complementary for a given target environment (target-traits). Under water-limited situations, traits that improve water 


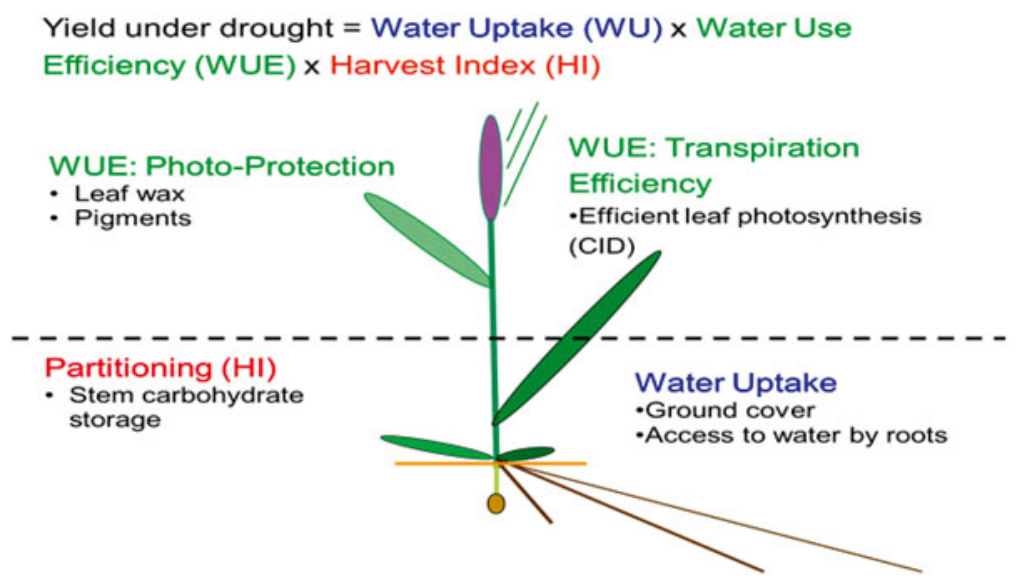

Fig. 41.4 Traits used in strategic crossing to combine complementary drought adaptive traits at CIMMYT since 2005

uptake, water use efficiency and partitioning to yield, respectively, are likely to work synergistically to maximize productivity (Richards 2006). Achieving this in a practical breeding context involves the following interventions, expressed chronologically:

(i) Target traits are identified using conceptual models as described above.

(ii) Genetic resources are screened to provide potential sources of target traits.

(iii) Candidate parents are characterized for traits that may contribute to genetic gains in target environments (Fig. 41.4).

(iv) Crosses are made such that traits expressed by respective parents encompass as many of the target-traits as possible. Top crosses may be used to facilitate the accumulation of traits.

(v) Early generation bulks are screened for integrative traits such as canopy temperature $(\mathrm{CT})$ in relevant environments; families with warm canopies -compared to checks- are mainly discarded.

The focus of much physiological research in the past has been to identify traits for early generation selection (EGS) that can be used to screen progeny for favorable trait expression. There is consensus that the more feasible traits are either genetically relatively simple, or integrative traits that explain a reasonable degree of the variation in yield and are relatively quick and easy to measure (Richards 2006). In fact very few traits meet these criteria and have found application. A successful application in wheat was the use of carbon isotope discrimination (CID) to select progeny with high transpiration efficiency conferring a conservative rate of water use that is associated with increased yield when the soil water profile is restricted (Richards 2006). Another trait that has found application in EGS under a range of 
environments is canopy temperature (CT). Under drought, selection for cooler CT permits genetic gains for yield (Olivares-Villegas et al. 2007) and genotypes with cooler canopies have been shown to extract more water from deeper soil profiles (Reynolds et al. 2007b). The trait is measured using infra-red thermometry and is ideal for high throughput screening for the following reasons (i) it is quick and easy to measure, (ii) the technology is inexpensive (currently US\$200 for a hand held instrument) and is amenable to airborne approaches as described above, (iii) CT shows robust association with performance; trait expression shows low interaction with growth stage or time of day. The trait is used routinely by CIMMYT's wheat breeding program for rainfed environments to enrich for alleles associated with dehydration resistance. For example, $\mathrm{F}_{3}$ and $\mathrm{F}_{4}$ bulks are screened for $\mathrm{CT}$ under drought; a larger number of plants -also expressing favourable agronomic traits- are selected from cooler CT families while bulks with warmer CT (compared to checks) are discarded. The development of relatively easy to use spectral radiometers offers another high throughput screening approach for comparing spectral reflectance indices (SRIs) of genotypes.

The result of investment in physiological trait (PT) based crossing has generated advanced lines distributed by CIMMYT as part of the 23rd Semi Arid Wheat Screening Nursery -SAWSN- and the 17th. Semi Arid Wheat Yield Trial (17th SAWYT). Of the 205 candidate genotypes for SAWSN in 2009, 48 (23\%) were derived from PT crossing and a similar proportion were represented in the SAWYT. The PT lines performed well in 2010 with the average yield of the PT lines beating the group of conventionally bred lines at $75 \%$ of the international sites. The most recent products of physiological breeding have shown spectacular results, with $70 \%$ of newest PT (PT-SA) lines beating the drought adapted check Vorobey under drought in Mexico in 2012, some by as much as $30 \%$. The underlying assumption for the PT strategy is that crosses between parents with different but potentially complementary PT expression will realize cumulative gene action in selected progeny. This has been borne out in previous analyses (Reynolds et al. 2009) and was shown again in the newest PT-SA lines which have landraces, re-synthesized hexaploids, and early PT lines in their pedigrees (Fig. 41.5).

\section{Conclusions}

Phenotyping for expression of physiological traits is an efficient way to select among genetic resources for promising candidate parents that can be used to accumulate stress adaptive traits and alleles. High throughput phenotyping approaches can facilitate the process of screening and progeny selection as well as the characterization of mapping populations. Genetic analysis of the latter will ultimately lead to identification of molecular markers that can be used in breeding and mining genetic resources for allelic diversity. 
a

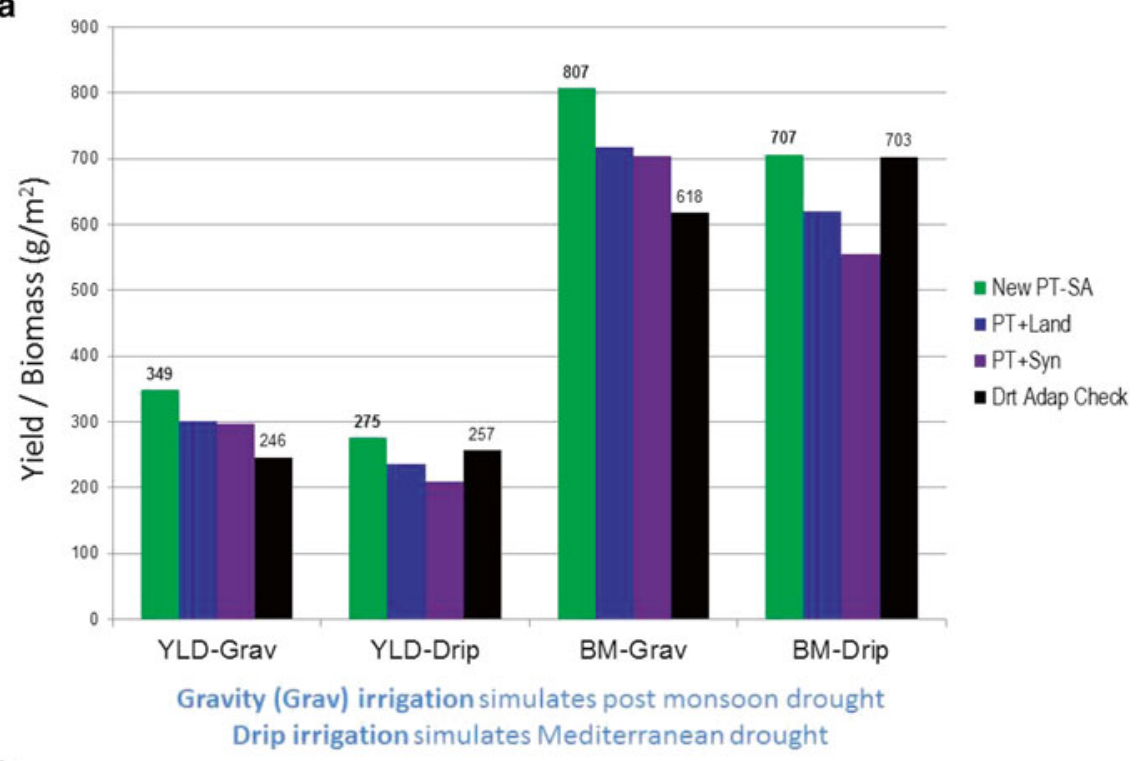

b

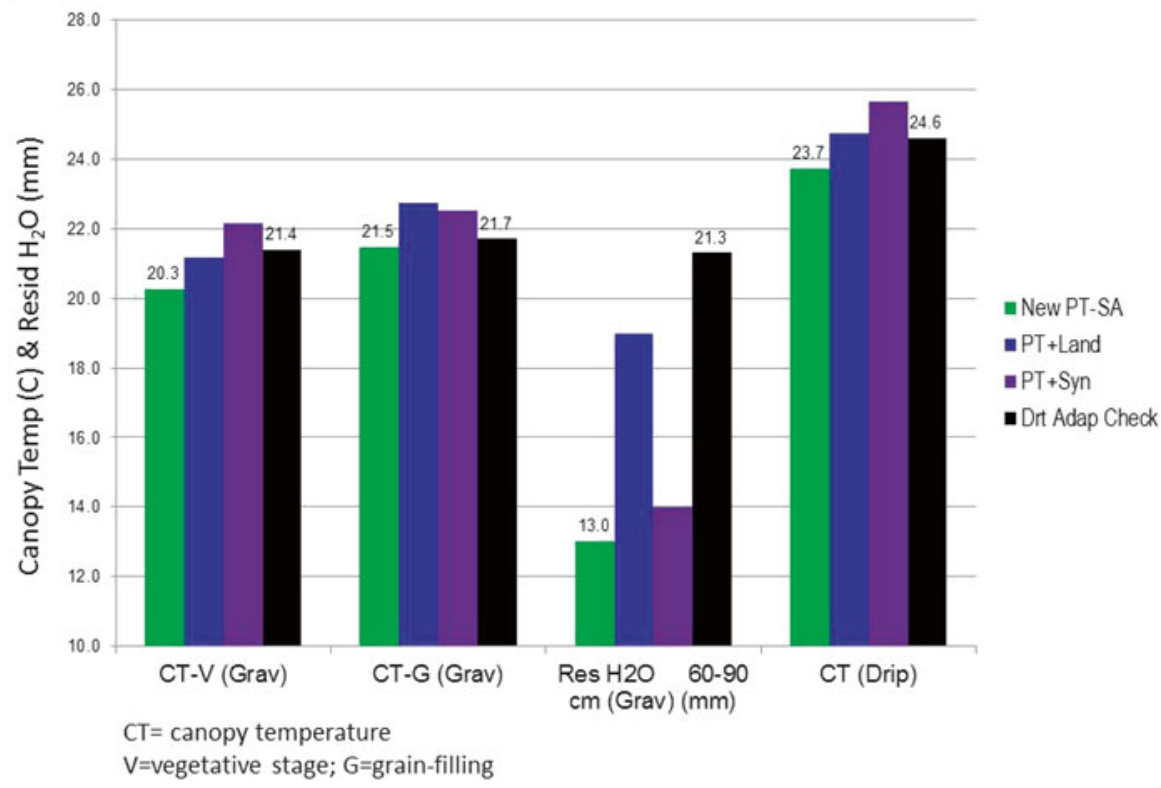

Fig. 41.5 New PT-SA line SOKOLL/3/PASTOR//HXL7573/2*BAU/4/WBLL4//OAX93.24.35/ WBLL1 containing PT + synthetic (Sokoll) (PT + Syn) and PT + Mexican landrace (OAX93.24.35) $(\mathrm{PT}+\mathrm{Land})$ in its pedigree grown under two distinct drought environments: (a) yield and biomass under gravity and drip irrigation simulating post monsoon stored soil moisture, and Mediterranean drought environments, respectively. (b) Improved water relations in new PT line showing canopy temperature and residual soil moisture at harvest. Traits are compared with the drought adapted check Vorobey (also with synthetic in its background) 
Open Access This chapter is distributed under the terms of the Creative Commons Attribution Noncommercial License, which permits any noncommercial use, distribution, and reproduction in any medium, provided the original author(s) and source are credited.

\section{References}

Aparicio N, Villegas D, Casadesus J et al (2000) Spectral vegetation indices as nondestructive tools for determining durum wheat yield. Agron J 92:83

Berni J, Zarco-Tejada PJ, Suarez L, Fereres E (2009) Thermal and narrowband multispectral remote sensing for vegetation monitoring from an unmanned aerial vehicle. IEEE Trans Geosci Remote Sens 47:722-738

Braun HJ, Atlin G, Payne T (2010) Multi-location testing as a tool to identify plant response to global climate change. In: Reynolds MP (ed) Climate change and crop production, CABI climate change series. CABI, Wallingford, pp 115-138

Cossani CM, Reynolds MP (2012) Physiological traits for improving heat tolerance in wheat. Plant Physiol 160:1710-1718

Gourdji SM, Mathews KL, Reynolds MP et al (2012) An assessment of wheat breeding gains in hot environments. Proc R Soc B Biol Sci 280:1752-1760

Hajjar R, Hodgkin T (2007) The use of wild relatives in crop improvement: a survey of developments over the last 20 years. Euphytica 156:1-13

Jones CL, Weckler PR, Maness NO et al (2007) Remote sensing to estimate chlorophyll concentration in spinach using multi-spectral plant reflectance. Am Soc Agric Biol Eng 50:2267-2273

Leinonen I, Jones HG (2004) Combining thermal and visible imagery for estimating canopy temperature and identifying plant stress. J Exp Bot 55:1423-1431

Lopes M, Reynolds MP (2011) Drought adaptive traits and wide adaptation in elite lines derived from re-synthesized hexaploid wheat. Crop Sci 51:1617

Manes Y, Gomez HF, Puhl L et al (2012) Genetic yield gains of the CIMMYT international semiarid wheat yield trials from 1994 to 2010, 2012. Crop Sci 52:1543-1552

Mujeeb-Kazi A, Rosas V, Roldan S (1996) Conservation of the genetic variation of Triticum tauschii (Coss.) Schmalh. (Aegilops squarrosa auct. non L.) in synthetic hexaploid wheats (T. turgidum L. s.lat. $\times$ T. tauschii; $2 \mathrm{n}=6 \times=42$, AABBDD) and its potential utilization for wheat improvement. Genet Resour Crop Evol 43:129-134

Nakashima K, Ito Y, Yamaguchi-Shinozaki K (2009) Transcriptional regulatory networks in response to abiotic stresses in Arabidopsis and grasses. Plant Physiol 149:88-95

Olivares-Villegas JJ, Reynolds MP, McDonald GK (2007) Drought-adaptive attributes in the Seri/ Babax hexaploid wheat population. Funct Plant Biol 34:189-203

Qin F, Shinozaki K, Yamaguchi-Shinozaki K (2011) Achievements and challenges in understanding plant abiotic stress responses and tolerance. Plant Cell Physiol 52:1569-1582

Rebetzke GJ, Chapman SC, Mcintyre L et al (2009) Grain yield improvement in water-limited environments. In: Carve BF (ed) Wheat: science and trade. Ames, Wiley-Blackwell, pp 215-249

Reynolds MP, Borlaug NE (2006) Impacts of breeding on international collaborative wheat improvement. J Agric Sci 144:3-17

Reynolds M, Balota M, Delgado M et al (1994) Physiological and morphological traits associated with spring wheat yield under hot, irrigated conditions. Aust J Plant Physiol 21:717

Reynolds MP, Saint Pierre C, Abu SIS et al (2007a) Evaluating potential genetic gains in wheat associated with stress-adaptive trait expression in diverse germplasm under drought and heat stress. Crop Sci 47:172-189

Reynolds MP, Dreccer F, Trethowan R (2007b) Drought adaptive traits derived from wheat wild relatives and landraces. J Exp Bot 58:177-186 
Reynolds MP, Manes Y, Izanloo A, Langridge P (2009) Phenotyping for physiological breeding and gene discovery in wheat. Ann Appl Biol 155:309-320

Reynolds MP, Hays D, Chapman S (2010) Breeding for adaptation to heat and drought stress. In: Reynolds MP (ed) Climate change and crop production, vol 1, CABI climate change series. CABI, Oxfordshire

Richards RA (2006) Physiological traits used in the breeding of new cultivars for water-scarce environments. Agric Water Manag 80:197-211

Rosegrant MW, Agcaoili M (2010) Global food demand, supply, and price prospects to 2010. International Food Policy Research Institute, Washington, DC

Saint Pierre C, Crossa JL, Bonnett D et al (2012) Phenotyping transgenic wheat for drought resistance. J Exp Bot 63:1799-1808

Sharma RC, Crossa J, Velu G et al (2012) Genetic gains for grain yield in CIMMYT spring bread wheat across international environments. Crop Sci 52:1522-1533

Smale M, Reynolds M, Warburton M et al (2002) Dimensions of diversity in CIMMYT bread wheat from 1965 to the present. Crop Sci 42:1766-1779

Tattaris M, Reynolds M, Pietragalla J et al (2013) Airborne remote sensing for high throughput phenotyping of wheat. In: Proceedings of the workshop UAV-based remote sensing methods for monitoring vegetation, Cologne

Trethowan RM, Mujeeb-Kazi A (2008) Novel germplasm resources for improving environmental stress tolerance of hexaploid wheat. Crop Sci 48:1255-1265 